Objective To investigate the pattern of sleep and its relationship with obesity in primary school children.

Materials and Methods We carried out a cross sectional study. Data were obtained from a representative sample 270 primary school children (119 boys and 151girls) aged 6-9 years. Sleep pattern was measured for 7 consecutive days using Actigraph accelerometer as well as daily logs. Directly measured height and weight data were used to define overweight and obesity according to the centers for disease Control and Prevention's (CDC) 2000 Growth Charts.

Results No significant difference ( $p>0.05)$ was found in current study between boys and girls for BMI percentiles, weekend sleep duration and efficiency. But girls were found to have higher values $(p<0.05)$ than those of boys for height, weight for age and lower values for 7 days and weekdays sleep duration. However, after adjusting these data for age the significant difference for 7 days and weekdays sleep duration disappeared. In addition, sleep efficiency was higher $(p<0.05)$ in girls than boys during 7 days and weekdays.

Conclusions The current study revealed no differences for sleep duration and efficiency among overweight and obese children compared to normal weight one. However, other lifestyle factors might be related to body weight status in children.

\section{RELATIONS BETWEEN OBESITY AND 25-HYDROXYVITAMIN D IN EMIRATI YOUTH}

doi:10.1136/archdischild-2012-302724.1426

'SM Shah, ${ }^{2} \mathrm{~S}$ Muhairi, ${ }^{2} \mathrm{M}$ Naqbi, ${ }^{2} \mathrm{~A}$ Khouri, ${ }^{2} \mathrm{~A}$ Mehairi, ${ }^{3} \mathrm{~F}$ Al Maskari. ${ }^{1}$ Faculty of Medicine and Health Sciences; ${ }^{2}$ Family Medicine; ${ }^{3}$ Community Medicine, United Arab Emirates University, FMHS, Al Ain, United Arab Emirates

Objectives The aim of this study was to identify the correlation of obesity with 25-hydroxyvitamin D levels in a population-based sample of schoolchildren Al Ain, United Arab Emirates.

Methods It was a cross-sectional study. A random sample of 1018 adolescents aged 12 to 18 years was selected from 140 Schools. A self-administered questionnaire was used to obtain socio-demographic characteristics, physical activity and dietary habits. Blood samples were collected after overnight fast for more than 8 hours. Blood pressure (BP), height weight, waist and hip circumference measurements were made by trained nurses. Body mass index (BMI) less than $85^{\text {th }}$, greater than $85^{\text {th }}$ but less than $95^{\text {th }}$, and $\geq 95^{\text {th }}$ percentiles for age and gender were used to define normal, overweight and obese according to the 2000 CDC growth charts. Fasting blood glucose and plasma lipids were also measured. Serum $25(\mathrm{OH}) \mathrm{D}$ concentrations were measured in subset of youth aged 15 to 18 years.

Results The age of study participants ( $n=315)$ ranged from 15 to 18 years. Overall 13\% of the participants had vitamin D deficiency $(<15 \mathrm{ng} / \mathrm{mL})$. A high proportion (21\%) of female youth had vitamin $\mathrm{D}$ deficiency compared to their male counterparts (5\%). After adjustment for age, female gender, body mass index was negatively correlated with vitamin D levels $(\mathrm{p}<0.05)$.

Conclusion The present findings emphasize the importance of obesity prevention in order to prevent vitamin $\mathrm{D}$ defiance. There is significant disparity by gender and vitamin $\mathrm{D}$ deficiency prevention programs should particular focus female children.

\section{FAST-FOOD CONSUMPTION, MICRONUTRIENT INTAKE AND ANXIETY LEVELS AMONG PUPILS AGED 8-12 YEARS OLD IN TEHRAN-IRAN}

doi:10.1136/archdischild-2012-302724.1427

M Bakhtiyari. Tehran University of Medical Sciences, Tehran, Iran

Background and Aims With regard to excessive consumption of fast foods as an indicator of a lifestyle characterized by urbanization, overworking, low resting time, insufficient time for choosing or preparing food and lack of a pertinent study about this issue in Iran, the present study was conducted aiming at analyzing the relationship between consumption of fast foods and the amount of micronutrient intake as well as anxiety.

Methods We conducted a cross-section study with 488 pupils aged 8-12 years living in Tehran selected by cluster sampling from 22 districts of Tehran. Diet assessments were done using 24 hours recall in two times with a week interval. Levels of anxiety were determined using the Persian version of the validated Speilburger test. A regression analysis model with appropriate odds was used to assess the effect of fast food consumption on anxiety variables.

Results Significant statistical relationship was observed between consumption amount of fast foods and trait and state anxiety levels. Adjusting for age, gender and body mass index, the Proportional Odds Regression Model showed a significant relationship between consumption amount of fast foods and trait and state anxiety. odds of getting either moderate or severe anxiety in those students who frequently consumed fast foods was seven folds higher than those students who rarely used fast foods.

Conclusions High consumption of Fast-foods was associated with the increased anxiety levels.

\section{ASSOCIATION BETWEEN VISCERAL FAT AND BLO0D PRESSURE IN YOUNG ADOLESCENTS IN WESTERN INDIA}

doi:10.1136/archdischild-2012-302724.1428

${ }^{1,2}$ AS Nimbalkar, ${ }^{2}$ SS Singh, ${ }^{2}$ SR Singh, 1,3SM Nimbalkar. ${ }^{1}$ Department of Pediatrics; ${ }^{2}$ Department of Physiology, Pramukhswami Medical College; ${ }^{3}$ Central Research Services, Charutar Arogya Mandal, Anand, India

Background and Aims Risk of metabolic syndrome is 5 times higher in adolescents with more visceral fat deposition. We studied the association between visceral fat and blood pressure in young adolescents of gujarati ethnicity.

Methods 50 young healthy adolescents (age 17 to 21 years) of both genders from local community college participated in the crosssectional non-experimental study. Omron Body Composition Monitor was used to quantify visceral fat level and visceral fat analysis. Height, Blood Pressure, Weight and other obesity parameters were measured. Results were expressed as mean $\pm S D$. Independent samples $\mathrm{T}$ test and Linear regression analysis was used.

Results Boys and Girls were equally recruited. No significant differences found between groups for pulse, diastolic pressure (DP) and visceral fat (VF) but differences between boys and girls for systolic pressure (SP), pulse pressure (PP) and mean arterial pressure (MAP) were present. Significant correlation between Visceral Fat $(2.54 \pm 2.2)$ and Systolic Pressure (102.88 \pm 9.99$)$, Diastolic Pressure (69 \pm 8.23 ), Mean Arterial Pressure (80.26 \pm 8.53$)$ and Pulse Pressure (33.88 \pm 4.98$)$ for females and between Visceral Fat $(4.8 \pm 3.74)$ and Systolic Pres-

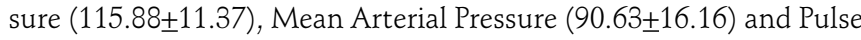
Pressure $(41.60+8.34)$ with the best correlation between Mean arterial Pressure and Visceral fat among males.

Conclusion Visceral obesity correlated with mean arterial pressure in both sexes with better correlation in males. Distribution of fat in the body is an important indicator which needs to be monitored and/or targeted in management of overweight and obese adolescents.

\section{LOWER COGNITIVE PERFORMANCE AMONG OBESE EGYPTIAN ADOLESCENTS}

doi:10.1136/archdischild-2012-302724.1429

${ }^{1}$ A Eladl, ${ }^{20}$ Eladl, ${ }^{3} \mathrm{H}$ Atwa. ${ }^{1}$ Especial Education, Post Graduate College, Arabian Gulf University, Manama; ${ }^{2}$ Misr International University; ${ }^{3}$ Pediatric, Faculty of Medicine, Suez Canal University, Ismailia, Egypt 